\title{
A Patient-specific Knee Joint Computer Model Using MRI Data and 'in vivo' Compressive Load from the Optical Force Measuring System
}

\author{
Božidar Potočnik ${ }^{1}$, Damjan Zazula ${ }^{1}$, Boris Cigale $^{1}$, Dušan Heric ${ }^{1}$, Edvard \\ Cibula $^{1}$ and Tomaž Tomažič ${ }^{2}$ \\ ${ }^{1}$ Faculty of Electrical Engineering and Computer Science, University of Maribor, Slovenia \\ ${ }^{2}$ University Clinical Centre of Maribor, University of Maribor, Slovenia
}

Modelling of patient knee joint from the MRI data and simulating its kinematics is presented. A flexion of the femur with respect to the tibia from $0^{\circ}$ to around $40^{\circ}$ is simulated. The finite element knee model is driven by compressive load measured 'in vivo' during MRI process by using specially developed optical force measurement system. Predicted kinematics is evaluated against the high-quality model obtained by registration from experimentally gathered low-quality MRI at fixed flexions. Validation pointed out that the mean square error (MSE) for the Euler rotation angles are bellow $1.73^{\circ}$, while the MSE for Euler translation is smaller than $5.93 \mathrm{~mm}$.

Keywords: compressive load, computer model, finite elements, knee joint, optical force sensor

\section{Introduction}

The knee joint is the largest and heavily loaded joint in the human body. Figure 1 depicts the knee joint with three main bones: bone femur, bone tibia, and bone patella. Consequently, the knee joint is highly susceptible to incidences of injuries and osteoarthrosis. Knowledge of 'in vivo' joint motion and loading during functional activities is, therefore needed to improve our understanding of knee joint degeneration and restoration. Such system for knee joint kinematics analysis and/or simulation should be able to deal with specificity of particular individuals. One possibility is motion analysis systems which expand our understanding of the mechanics of normal and pathological human movement (Rowe et al., 2000). Another possibility is human knee computer models, which also present an effective way of evaluating these characteristics during the design phase, and provide an indication of expected clinical performance (Bei and Fregly, 2004; Halloran et al., 2005).

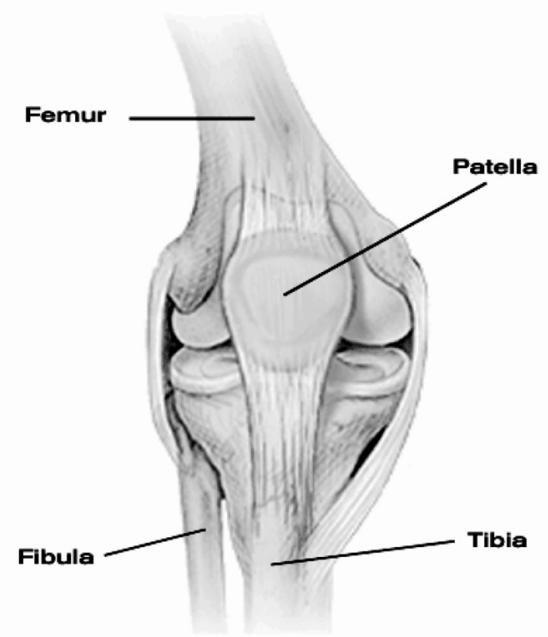

Figure 1. Human knee joint with main bones.

Motion analysis/measuring systems provide 'in vivo' joint motion data during functional activities. Current measuring techniques can be categorized into the following groups: 1) analysis of 2D radiographs, 2) 3D stereophotogrammetry, 
3) evaluating the movement of markers attached to the skin, 4) evaluation of external markers invasively attached to the bone, 5) cadaveric dissection studies, 6) 2D fluoroscopic motion measurement using bone models, and 7) evaluation of 2D images from computed tomography $(\mathrm{CT})$ and magnetic resonance imaging (MRI) (Patel et al., 2004; Piazza and Cavanagh, 2000; Freeman and Piskernikova, 2005). Measurement methods using markers attached to the skin or bone-implanted markers have been proven to be accurate enough to collect slow 'in vivo' knee joint dynamics (Beillas et al., 2004; Rowe et al. 2000; Jan et al., 2002; Schuler et al., 2005; Zhou et al., 2002). However, devices attached directly to the skin (e.g. optical skin markers) can incur errors due to relative motion between the skin and the bone. Transcutaneous bone pins can loosen, bend and/or interfere with normal muscle action (Schuler et al., 2005). Roentgen stereophotogrammetric analysis, and MR/CT imaging are normally gathered quasi-statically due to equipment limitations, and, thus, do not permit dynamic analysis. This can partially be overcome by using an open MR scanner. Several studies measuring kinematics indirectly from MRI data while a knee was loaded with constant weight have been recently reported (Patel et al., 2004; Rothe et al., 2004). The 2D fluoroscopic motion measurement methods have clear advantages as they do not need markers and enable a direct measurement of bone motion, however, the main drawback is the need for radiation.

A number of computer models, recently finite element (FE) models, have been developed to study knee joint mechanics (Halloran et al., 2005). These models are usually based on a $3 \mathrm{D}$ reconstruction of the knee joint from some modality imaging data (e.g. MRI or CT) or special 3D laser coordinate digitizing system (e.g. in Donahue et al., 2003). Additional data, such as material properties are then used to supplement these models. Some functional activities, e.g. full gait cycle in (Godest et al., 2002) or one-legged forward hopping in (Beillas et al., 2004), are then simulated, and the knee joint responses in terms of kinematics and pressure data are obtained. The simulation tools used are either commercial such as PAM-SAFE (ESI Group, Paris, France) or ABAQUS (ABAQUS Inc.,USA) (Bei and Fregly, 2004; Halloran et al., 2005; Godest et al., 2002) or specially designed numerical problem solvers (AbdelRahman and Hefzy, 1998). The quality of the predictions made by these models is largely dependent on the quality of the experimental data (e.g. loads) used to drive them (Beillas et al., 2004). FE models are usually evaluated against some 'in vitro' data from other studies, experimental data from kinematics measuring systems or, recently, 'in vivo' kinematics data (e.g. Beillas et al., 2004). A recent attempt at real-time model simulation is reported in (Jan et al., 2002), where this method actually visualizes, rather than just simulates, a 3D joint model driven by experimental kinematics data.

To the best of our knowledge, only a few computer models exist, based on actual 'in vivo' patient's data, i.e. anatomy and specific 'in vivo' kinematics. The first such approach is revealed in (Beillas et al., 2004), where a quality FE knee model of a male patient performing a onelegged forward hopping trail was constructed. The kinematics data driving this model was obtained during hopping by an 'in vivo' motion measuring system.

Our paper follows and upgrades the idea of 'in vivo' patient-specific knee joint modelling. There are two basic motives for this: firstly, to enable the simulations of knee-joint kinematics based only on sparse, fixed-flexion measurement data with no need for dynamic MRI, and secondly, to generalize and speed up the knee modelling procedure significantly. A methodology is presented for constructing a 3D FE knee joint model from patient-specific MRI data. A knee flexion from $0^{\circ}$ to around $40^{\circ}$ is simulated. The constructed model is driven by compressive load data measured 'in vivo' during patient imaging process at a few fixed-flexion positions whilst the knee is moderately loaded. A special optical force measuring system was developed for this task. The predictions, in terms of knee kinematics, made by this model were evaluated against experimentally gathered data obtained from a few fixed-flexion scans. Special evaluation procedures were developed for this task. All aspects of this study were approved by our local ethics committee, and written informed consent was obtained from all volunteers prior to MR imaging. The preliminary results and the basic modelling concept were reported in (Potocnik et al., 2005). In this work, a system for force measurement in a magnetic 
field was refined and accurately validated. Minor modifications were also done in template knee-joint FE model, especially by model of MR exercise rig. The material and structural properties of knee joint structures were studied in greater detail once again. Some parameters were, consequently, fine tuned. Proposed modelling approach was thoroughly assessed on knee-joints of two patients. Both knee-joints were fully modelled and simulated by using patient-specific MRI data. Quantitative measures were introduced to assess this computer model quality. A special procedure for determining the Euler translation error was developed as well.

\section{Experimental Methods}

\subsection{Patient Imaging Data Acquisition}

Two male volunteers were examined (aged 22 and 52 years), having signs on meniscus or ligament tear. Imaging material was acquired using a traditional 1.5 T MR scanner (Visart, Toshiba, Tokyo, Japan). A knee joint of each patient was scanned twice, namely: 1) with high-quality static MR protocol and 2) with low-quality static MR protocol repeated at a few different fixed knee flexions.

High-quality static MR protocol was established to ease the 3D reconstruction of the patient's knee joint. This protocol uses a FE3D image technique with the quadrature (QD) knee coil of an MR scanner (TR $41 \mathrm{~ms}$, TE $9 \mathrm{~ms}$, flip angle 18/73, NAQ 1). Image acquisition was performed in sagittal orientation with $2 \mathrm{~mm}$ slice thickness. The field of view (FOV) was $22 \times 22$ $\mathrm{cm}$ with in-plane resolution of $0.43 \mathrm{~mm}$ (output image matrix was 512 x 512 pixels). Acquisition time was around 22 minutes for a sequence consisting of 60 slices (cross-sections). The patient's knee was slightly supported during static MR protocol, thus provoking approximately $10^{\circ}$ knee flexion.

The purpose of acquiring a sparse sequence of MR images at a few fixed flexions was to permit the evaluation of the kinematic behaviour of the FE knee joint model. A patient with a flexed knee exerts a light force by pushing on a foot pedal during this imaging protocol (see Figure $2 b$ ). The MR data and 'in vivo' compressive load data are acquired simultaneously. The patient is scanned for up to 6 different knee flexion positions. This protocol uses a FE2D image technique with the joint pair knee coil of an MR scanner (TR $245 \mathrm{~ms}$, TE $15 \mathrm{~ms}$, flip angle 90, NAQ 1, gap 0.8). Only 9 slices of $4.3 \mathrm{~mm}$ thicknesses were acquired in sagittal orientation at each knee flexion position. The FOV was $17 \times 17 \mathrm{~cm}$ with an in-plane resolution of $0.66 \mathrm{~mm}$ (the output image matrix was $256 \times 256$ pixels). The acquisition time with this fast protocol was less than 2 minutes per flexion position.

A special MR compliant exercise rig was designed for this protocol (see Figure 2a, construction details are in (Simbio project, 2003)).

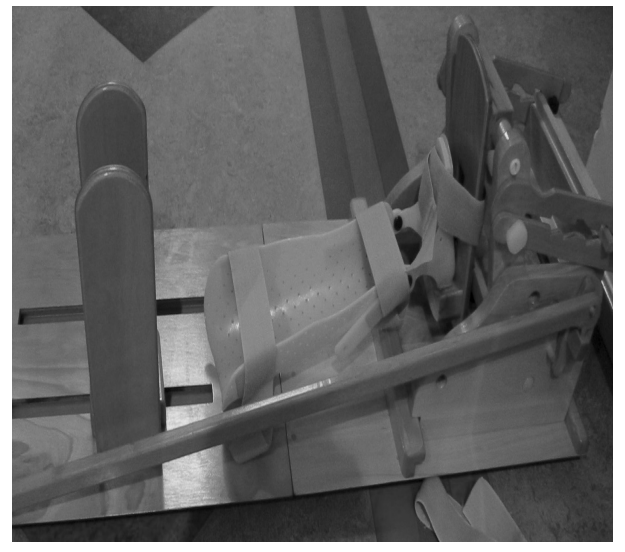

(a)

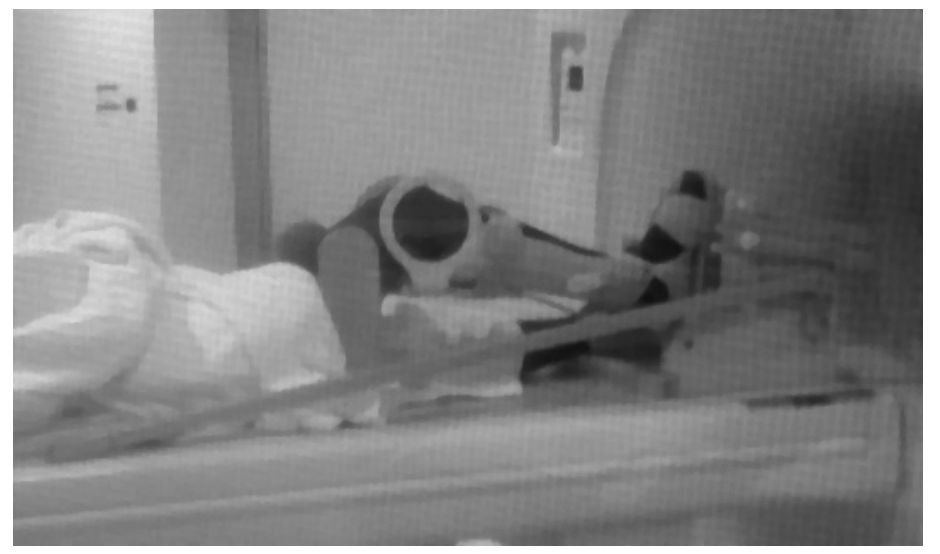

(b)

Figure 2. MR compliant exercise rig: a) basic components: wooden base and flexible ankle-foot orthosis integrated within the pedal, b) patient during scanning exerts light force to the pedal comprising the integrated force measuring system. 
The rig's basis is an adjustable, with respect to the patient's height, wooden board with a shoulder support (support is not shown). The two main parts are attached to the board: 1) a flexible ankle-foot orthosis for fastening shank and ankle, and 2) a wooden pedal. The pedal, on the one hand, enables the setting of 6 different knee flexion angles; on the other hand, it also measures the force (compressive load) the patient is exerting during imaging. Measurements are carried out by an integrated optical sensor. A flexion position can be selected manually by a small handle mounted on the pedal. Angles vary with respect to the patient's leg length but, generally, are in the range from $0^{\circ}$ to $60^{\circ}$ flexion with $8^{\circ}$ to $10^{\circ}$ increments.

\subsection{System for Force Measurement in a Magnetic Field}

When being scanned at predefined fixed knee flexions, the patient pushes his foot slightly against the pedal of the exercise rig. This compressive load on the foot is measured and, subsequently, used in the modelling phase. Therefore, a simple and fully electrical passive force measurement system was developed that can operate in those environments where magnetic fields exceed 1T. Special attention was devoted to the exclusive use of dielectric materials and, thus, the designed system does not induce any magnetic field distortions.

The presented force sensor design is based on displacement measurement by macrobend loss effect in single mode fibres (Gauthier and Ross, 1997; Donlagic and Culshaw, 2000; Gambling et al., 1978; Sharma et al., 1984). The main sensing element is a small fibre optic coil that is perturbed (squeezed) under applied force.

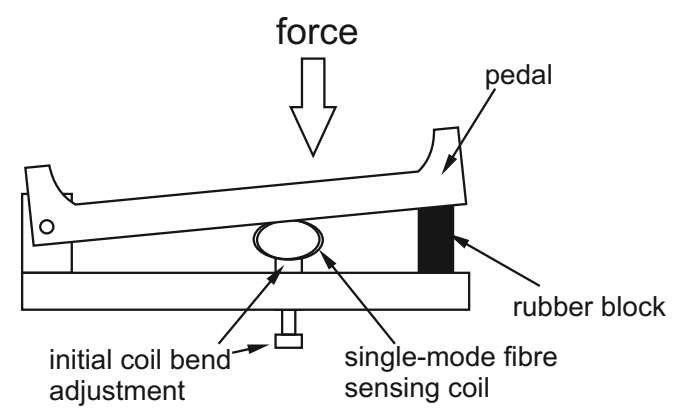

Figure 3. Mechanical configuration of the force sensor.
The practical mechanical design of the sensor is shown in Figure 3. The patient depresses the specially designed wooden pedal containing the sensing fiber coil. The elastic element (rubber block) was used between the pedal and the rest of the support structure, to convert the force asserted by the patient's foot to the pedal displacement. This displacement decreases a local bend radius of the fiber at horizontal edges of the sensing coil and thus increases the optical loss within the sensing coil (thereby it decreases intensity ratio of the light at the outputs of the sensing and reference fiber branches).

This system was calibrated to measure the normal component of the force asserted to the center of the pedal. The total range of the system was $0-240 \mathrm{~N}$, but other force ranges could be also covered by adjustment of the properties of the elastic element that converts force to displacement. The resolution was better than 0.5 N. After the calibration, the absolute accuracy of the sensor proved to be better than $\pm 7 \mathrm{~N}$. Measurements and calibration were performed at room temperature $(25 \pm 5 \mathrm{C})$ and relative humidity in the range from $40-60 \%$.

\section{Computational Methods}

\subsection{Image Processing and 3D Reconstruction}

A 3D knee joint is reconstructed from highquality static $2 \mathrm{D}$ patient MR imaging data by using non-linear registration, developed at the University of Sheffield during the SimBio project (Simbio project, 2003; Wood et al., 2002). Image registration was applied for two reasons: 1) the edges of the knee structures are weak, and 2) integration of prior knowledge is simple and efficient.

The core of this registration is a template (reference) knee mesh (see Figure 4a). This mesh is constructed only once from higher-quality static MR protocol and is immutable in the registration process. It is obtained by manually segmenting the knee structures on every slice and, afterwards, performing reconstruction from the obtained partial results. The slice sequence used 


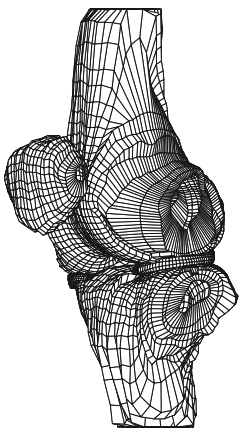

(a)

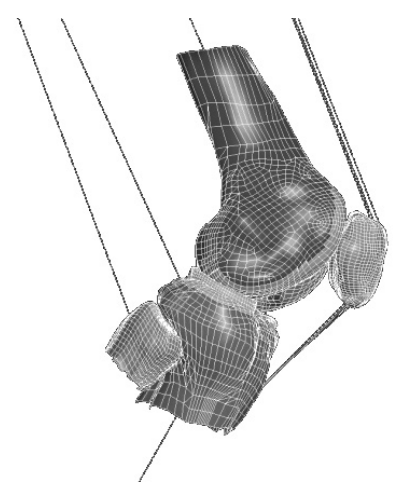

(b)

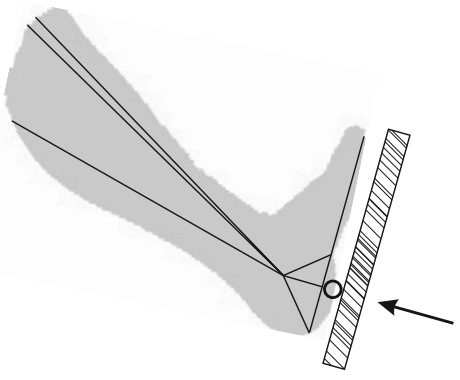

(c)

Figure 4. Knee joint model: a) template knee mesh consisting of eight structures: bones tibia, femur, patella and their corresponding cartilages, medial and lateral meniscus; b) patient-specific FE model; and c) lower leg and MR exercise rig model.

for the template knee mesh is denoted as template image. It was built from healthy malevolunteer knee data. The template knee mesh could generally be treated as the mesh of an average human knee (e.g. an averaged European knee).

Subsequently, the constructed template knee mesh is transformed/mapped into a patient 3D knee joint mesh based on the high-quality 2D patient MR data (patient image). The mapping function, having both a global and a local part, is determined by registration of the template image to patient image. A quality measure for goodness-of-fit between both images is based on the sum-of-squares of the differences in voxel grey-level intensities. The described registration and mapping of a template knee produce high-quality patient-specific 3D knee meshes. This reconstruction is completely automated.

\subsection{Template 3D Knee Joint FE Model}

The template 3D knee joint mesh beside bones, their cartilages and menisci comprises also other knee structures like e.g. ligaments and tendons. This template is actually the mesh from finite elements. The explicit FE code, PAM-SAFE ${ }^{\mathrm{TM}}$, is used in the modelling process. The final template mesh consisted of 3464 8-node hexahedral solid elements, 13120 shell elements, the majority of which were included as part of a rigid body and the remainder used in the contact interface definition, and $232 \mathrm{bar} /$ beam elements.
This template model was developed at the University of Sheffield during the SimBio project (Simbio project, 2003) based on their previous knee model (Penrose et al., 2002). Brief summary of this model will be given later in the text, including all minor model modifications carried out in this work.

Let us recapitulate the template properties. Three main knee bones, i.e. femur, tibia and patella, and part of the fibula are defined as rigid bodies to avoid deformations. For the same reason, the lateral and medial menisci are rigid bodies, as well. These bodies are in contact, thus defining contact surfaces. Seven such contacts are in our model: femur-patella, femurmeniscus, tibia-meniscus, and femur-tibia (two anterior and two posterior contacts). For each contact 'master' and 'slave' surfaces were defined. The articular cartilages of bones are defined as elastic plastic solids. Bars are used to link the anterior and posterior horns of the menisci to the tibia-104 bars for the lateral meniscus and 90 for the medial meniscus. A bar is a special 1D non-linear tension-only element defined by two nodes and some material properties.

Ligaments and muscles are used to define the interdependence of bones and, consequently, enable knee kinematics. The stability of the knee is also ensured by anterior crucial ligaments $(6$ bars) and posterior cruciate ligaments ( 9 bars). Lateral and medial collateral ligaments are defined by 4 bars on each side of the knee. They are the main links between the femur and tibia (or fibula). Quadriceps' muscles and tendons 
are modelled by 3 bars. The quadriceps act as knee extensors and, thus, in order to flex the knee fully this muscle must be relaxed. Three bars represent the three main vastii (lateralis, medialis, and intermedius) that form the quadriceps' muscles (Simbio project, 2003). They were permitted to elongate as a function of time during simulation. The patellar ligament, consisting of 5 bars, connects the patella to the anterior of the tibia. It is a continuation of the quadriceps' muscles and tendons. Hamstring is defined by 2 bars, where one bar is connected to the fibula and the other to the tibia. The motion of the hamstring and quadriceps' tendons are restricted to the sagittal plane. Attachment positions of the bars to bones were carefully determined by inspecting imaging material and discussions with orthopaedic surgeons.

A correct selection of the material and structural properties of knee joint structures is crucial for successful modelling and simulation. It is well known that many of these properties depend on the patient and are also subject to temperature alterations. In this 'in vivo' study, the majority of patient-specific parameters could not be measured (e.g. pressure data on the tibia plateau, ligament strain), therefore, these parameters were selected from literature. The next simplification of our model is that all patient properties are the same as in the template. The material and structural properties used in the template model, gathered in Table 1, are in agreement with the data in (Beillas et al., 2004; Laasanen, 2003).

The described template knee joint model is actually mapped into the patient-specific 3D knee joint FE model as described in subsection 3.1 This process is completely automated, sometimes only the bar attachment positions need to be manually fixed/corrected. Figure $4 b$ depicts patient-specific FE model.

Model boundary conditions were estimated from patient-specific data. Several properties of shank and thigh were carefully measured for each patient (e.g. length, diameter, extent). Based on these measurements, a centre of gravity, mass, and three principle moments of inertia were estimated for bone femur and tibia according to (Zatsiorsky and Seluyanov, 1985). The whole leg was modelled to simulate a patient pushing against the pedal of the MR exercise rig during low-quality imaging. The missing parts of the femur and tibia bones are each modelled by 3 beams attached to the femoral and tibial bone segments. Estimated lengths and masses of the limb segments were used. These beams join in a common node. In the proximal node of the femur beam used to model the hip joint translations are prevented, however, rotations in all directions are allowed. In the distal node of tibia beam used to model the ankle joint only

a) Bones

\begin{tabular}{|c|c|c|}
\hline Density $\left(\mathrm{g} / \mathrm{cm}^{3}\right)$ & $\mathrm{E}(\mathrm{GPa})$ & $v$ \\
\hline \hline 2 & 1 & 0.3 \\
\hline
\end{tabular}

b) Meniscus and cartilages

\begin{tabular}{|l|c|c|c|c|c|}
\hline & Density $\left(\mathrm{g} / \mathrm{cm}^{3}\right)$ & $v$ & $\mathrm{G}(\mathrm{GPa})$ & $\mathrm{K}(\mathrm{GPa})$ & $\sigma(\mathrm{GPa})$ \\
\hline \hline Meniscus & 1 & 0.3 & 0.026 & 0.133 & 10 \\
Cartilage & 1 & 0.3 & 0.05 & 0.200 & 10 \\
\hline
\end{tabular}

c) Ligaments (ACL/PCL-anterior/posterior cruciate, LCL/MCL- lateral/medial collateral)

\begin{tabular}{|c|c|c|c|}
\hline & Density $\left(\mathrm{g} / \mathrm{cm}^{3}\right)$ & $\mathrm{k}(\mathrm{N} / \mathrm{mm})$ & $\epsilon$ \\
\hline \hline ACL/PCL & 1 & $200-260$ & 0.01 \\
LCL/MCL & 1 & $114-134$ & 0.02 \\
\hline
\end{tabular}

Table 1. The material and structural properties of the knee joint structures used in the template knee joint model. Young's modulus is denoted by E, Poisson's ratio by $v$, bulk modulus by K, short time shear modulus by G, yield stress by $\sigma$, elastic stiffness by $\mathrm{k}$, and strain by $\epsilon$. 
rotation in sagittal plane and translation in proximal/distal direction are allowed. Both major bones are free in all six degrees of freedom, while the motion of bone patella is restricted to the sagittal plane. The motion of medial/lateral meniscus anterior/posterior horns are free in all six degrees of freedom as well.

\subsection{Model of Exercise Rig and Kinematics/Loads}

A simple patient knee flexion was simulated by using the described FE model, taking into account the MR scanning procedure at preselected fixed flexions. Accordingly, a model of MR exercise rig with simplified foot-pedal structure was added (see Figure 4c).

The knee joint motion is controlled by several inputs. At the distal node of the tibia bar a compressive load is applied, measured 'in vivo' by the force measurement system (see section 2.2). This node is depicted in Figure 4c, where an arrow indicates the direction of the applied concentrated nodal load. Global loads are indirectly determined by the principle moments of inertia calculated for bones femur and tibia. Initial force effect or initial strain value for pretensioning is defined for ligaments ACL, LCL, MCL, and deep fibres MCL (see Table 1 for abbreviations). The loads of these element are ranging around 0.04, with the exception of the deep fibres MCL which are set to 0.13. Appropriate kinematic response of this model is attained also by suitable setting of linear elastic stiffness and mass per unit length of particular element (i.e. ligaments, quadriceps, and hamstrings). To conclude, we see that knee joint is actually modelled as a connected set of strings with single external force applied at the rig pedal. The simulator just seeks an equilibrium between system's outer and inner forces.

\subsection{Simulation}

The developed model simulates flexion of the knee by using an explicit FE code PAM-SAFE ${ }^{\mathrm{TM}}$. The following global settings were applied, namely quadratic bulk viscosity coefficient was fixed at 1.2, linear bulk viscosity coefficient was 0.06 , hourglass viscosity coefficient was 0.15 , and stiffness using elastic modulus was used for shell hourglass control. The FE simulation was performed in a $300 \mathrm{~ms}$ interval, usually finished in around 300 cycles (states). The time interval was $0.01 \mu$ s and the time step was set to 'small'.

Although our model comprises several knee structures, the motion of the femur bone with respect to the tibia bone was inspected in the present study only. Twenty out-of-plane nodes from each major bone were traced during simulation. The following $4 \times 20$ matrix was defined at the $i$-th cycle:

$$
\mathbf{X}_{i}=\left[\begin{array}{llll}
\mathbf{n}_{i, 1} & \mathbf{n}_{i, 2} & \ldots & \mathbf{n}_{i, 20}
\end{array}\right],
$$

where $\mathbf{n}_{i, j}=\left(x_{i, j}, y_{i, j}, z_{i, j}, 1\right)$ presents coordinates for the $j$-th node. Nodes at cycle 0 determine the initial bone positions. In the sequel, a transformation relative to the straight leg starting position was calculated, which was set as the zero flexion position (relative to the static knee flexion of around $10^{\circ}$ ). Therefore, the following overdetermined linear equation system is solved:

$$
\mathbf{X}_{i}=\mathbf{A}_{i} \mathbf{X}_{0},
$$

where $\mathbf{X}_{0}$ denotes the initial bone position, $\mathbf{X}_{i}$ denotes the bone position at cycle $i$, and $\mathbf{A}_{\mathbf{i}}$ denotes the affine matrix at cycle $i$. To capture the motion of the femur bone with respect to the tibia bone, a joint affine matrix $\mathbf{J}$ is calculated in $i$-th cycle as follows:

$$
\mathbf{J}_{\mathbf{i}}=\mathbf{T}_{\mathbf{i}}^{-1} \mathbf{F}_{\mathbf{i}}
$$

where $\mathbf{T}_{\mathbf{i}}$ and $\mathbf{F}_{\mathbf{i}}$ denote affine matrices for the tibia and femur bones, respectively. Affine matrices take the form of:

$$
\left[\begin{array}{rrrr} 
& {[\mathbf{R}]} & & {[\mathbf{L}]} \\
0 & 0 & 0 & 1
\end{array}\right]
$$

where $\mathbf{R}$ is $3 \times 3$ rotation matrix and $\mathbf{L}$ is the translation vector. Euler angles are the classic way of representing rotations in 3D Euclidean space. These rotation angles are designated in the medical domain as flexion/extension, internal/external rotation, and varus/valgus. The Euler angles relative to the initial knee position defined at cycle 0 are calculated from matrix $\mathbf{R}$ by using Levenberg-Marquardt optimisation algorithm (Press et al., 1992). Correspondingly, translations relative to the static knee position are calculated indirectly from vector $\mathbf{L}$. 


\subsection{Evaluation}

Simulation results were evaluated against patient imaging data obtained at preselected fixed knee flexions. The idea behind this evaluation is that bones are considered non-deformable structures, and, therefore, their structure and interdependence relationship to one another do not change over a short time period. Thus, if the same joint is scanned and reconstructed twice, then both $3 \mathrm{D}$ reconstructions must be equal, with just some possible misalignment. Let us present this idea in more detail. A partial (sparse) 3D volume is reconstructed from a small number of low-quality images (up to 9 sagittal slices). First, the bones femur and tibia were manually segmented by an expert. These segments were then stacked together to form a partial 3D volume. Sparse 3D volumes were constructed for both major bones at each flexion position (up to 6 flexion positions). Afterwards, each partial volume is registered to the appropriate 3D volume of the knee joint's major bone, reconstructed from the high-quality static imaging data. Figure 5 depicts a rigid registration example of partial 3D volume to the static 3D reconstructed volume for the femur bone. Figure 5 a depicts the situation before registration, while (b) depicts rigidly registered volumes. The rigid registration results in an affine transformation matrix. The Euler angles and translations are then calculated as described in subsection 3.4.

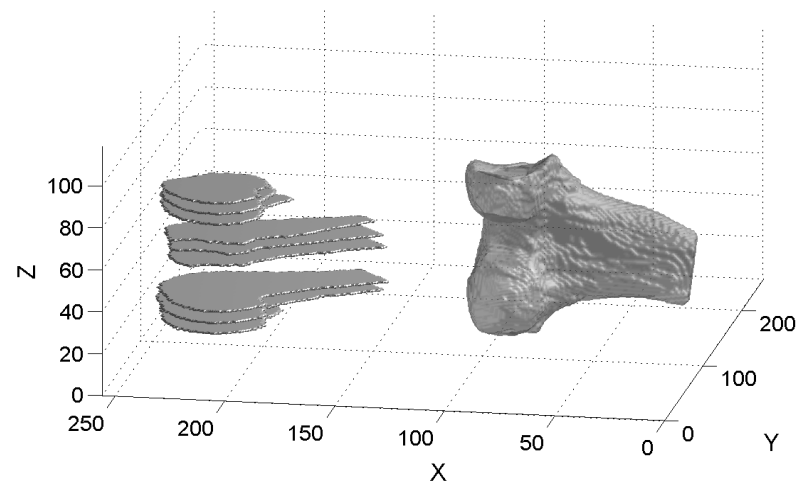

(a)

\section{Results}

The simulation of a patient-specific model results in the knee flexion from its initial extended position to the final position of approximately $40^{\circ}$. The average CPU time required to accomplish simulation was around 45 hours on a PC-based system with an Intel Pentium Xenon $2.2 \mathrm{GHz}$ processor and $1 \mathrm{~GB}$ RAM, where a mesh reconstruction took about 23 minutes and modelling around 1 hour of manual work. It should be noted that the most precise simulation with the lowest time step was selected in the PAM-SAFE ${ }^{\mathrm{TM}}$. This processing time can easily be reduced by distributed computing (Simbio project, 2003).

Results for two patients are presented in Figures 6 and 7. Both figures depict, in bold, the obtained simulation results versus flexion: (a) internal/external rotation, (b) varus/valgus rotation, (c) proximal/distal translation, (d) anterior/posterior translation, and (e) medial/lateral translation. Evaluation data are represented in the same figures by a dotted line. It should be noted that the first flexion angles (see evaluation data) appear as negative angles due to the patients having been scanned at around $10^{\circ}$ of static flexion. We have to stress again that all Euler angles and translations can only be calculated and reported versus flexion angle, separately for the experiment and simulation.

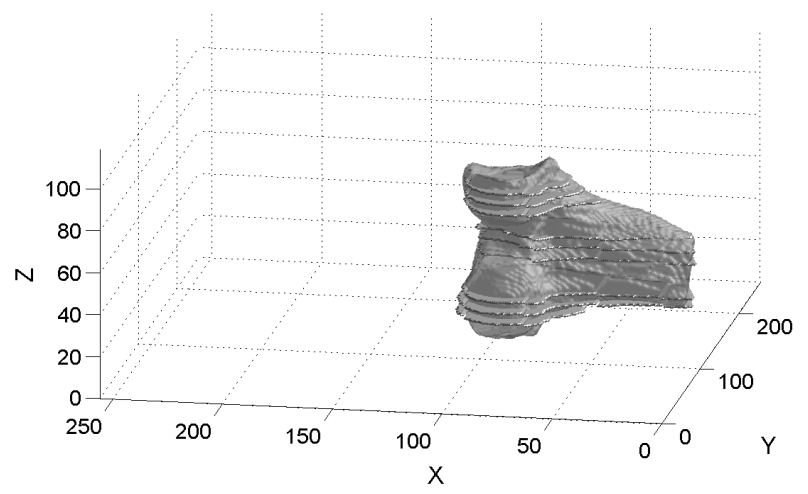

(b)

Figure 5. Rigid registration of a sparse, single fixed-flexion volume to the high-quality static volume (slices) for a femur bone: a) partial 3D volume and situation before registration, and b) situation after registration. 


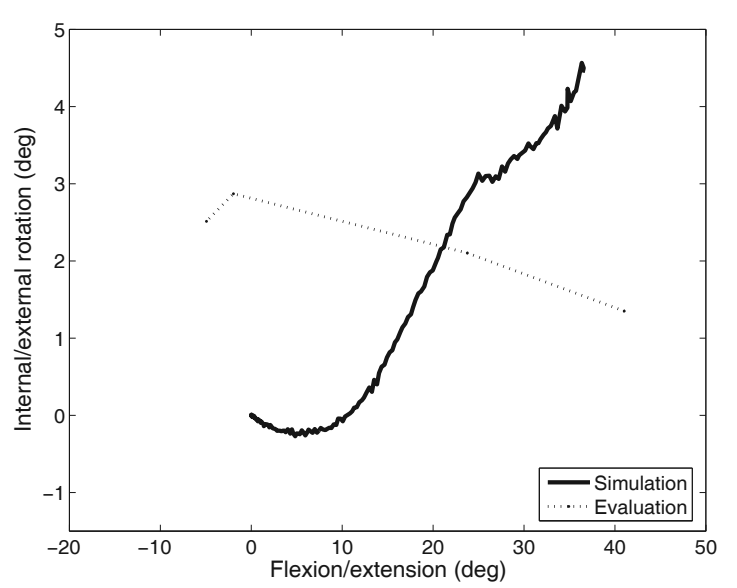

(a)

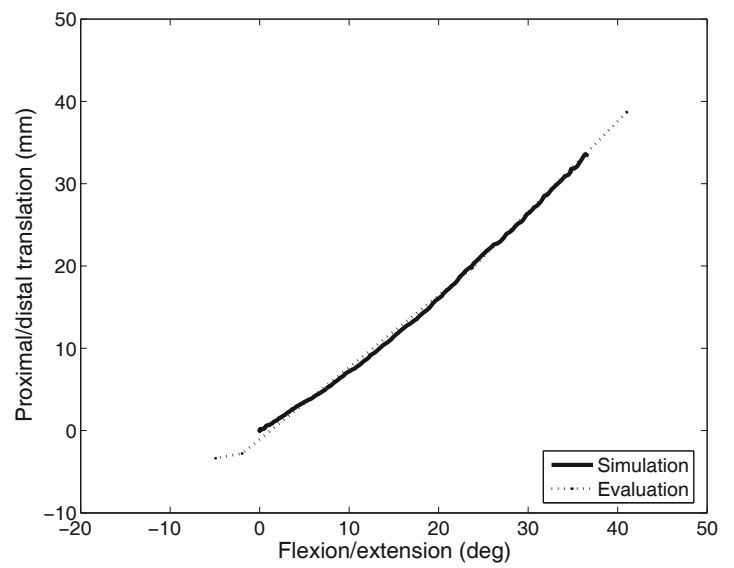

(c)

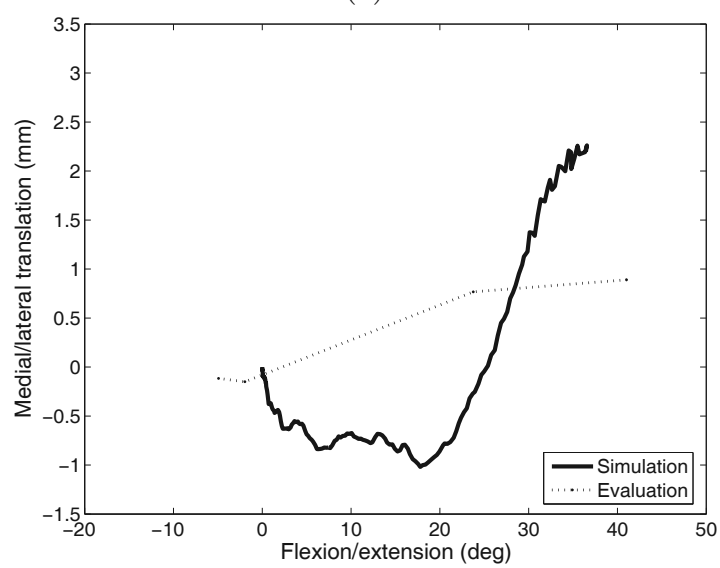

(e)

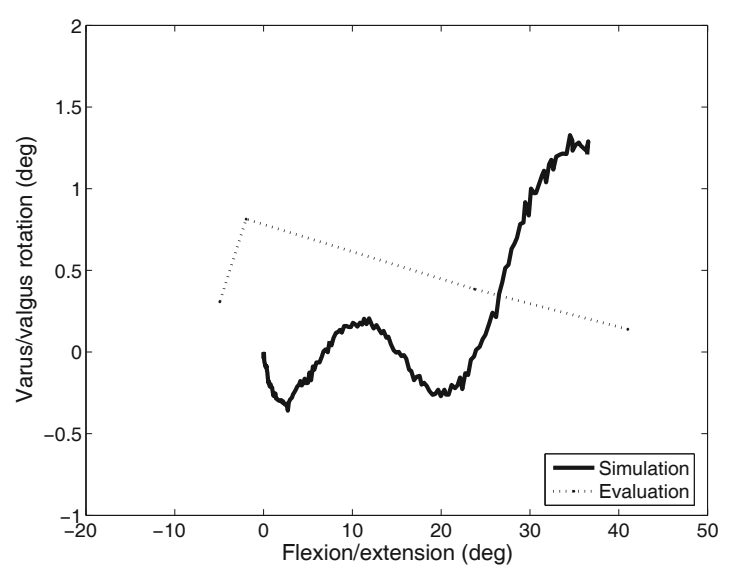

(b)

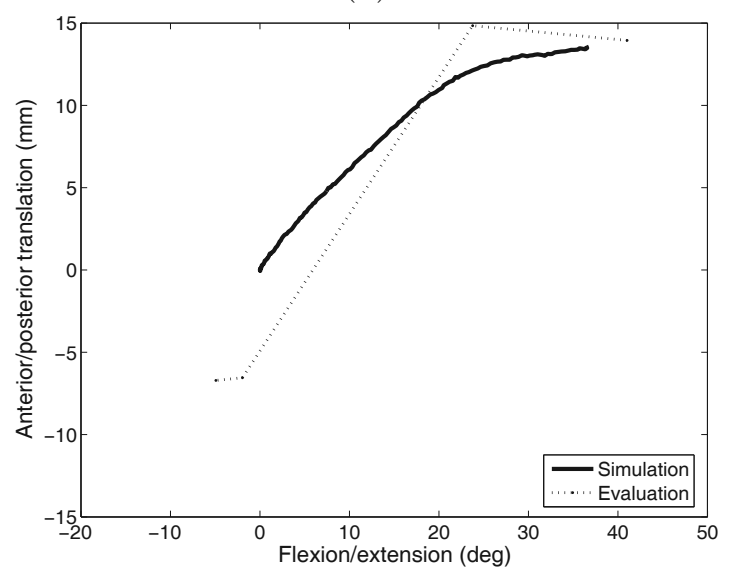

(d)

Figure 6. Simulation (bold) and experimental (dotted) results for patient A: a) internal/external rotation, b) varus/valgus rotation, c) proximal/distal translation, d) anterior/posterior translation, and e) medial/lateral translation. Results are depicted versus flexion angle.

A mean square error (MSE) between simulation and experimental data was calculated for all patients. The evaluation pointed out that average MSE for the internal/external rotation was $1.73^{\circ}$ (standard deviation $\mathrm{std}=0.1^{\circ}$ ), the average MSE for the varus/valgus rotation was $0.21^{\circ}\left(\mathrm{std}=0.03^{\circ}\right)$, the average MSE for the proximal/distal translation was $2.79 \mathrm{~mm}$ $(\mathrm{std}=2.68 \mathrm{~mm})$, the average MSE for the anterior/posterior translation was $5.93 \mathrm{~mm}(\mathrm{std}=1.41$ $\mathrm{mm}$ ), while the average MSE for the medial/lateral translation was $0.31 \mathrm{~mm}(\mathrm{std}=0.06 \mathrm{~mm})$. 


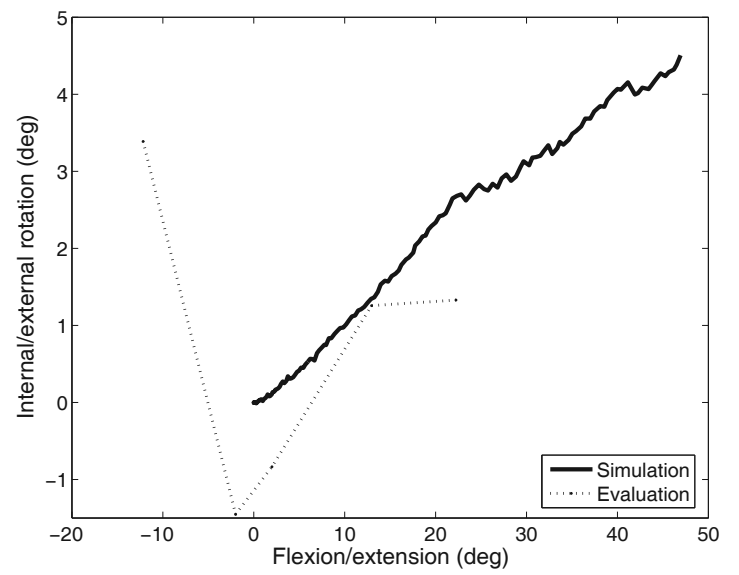

(a)

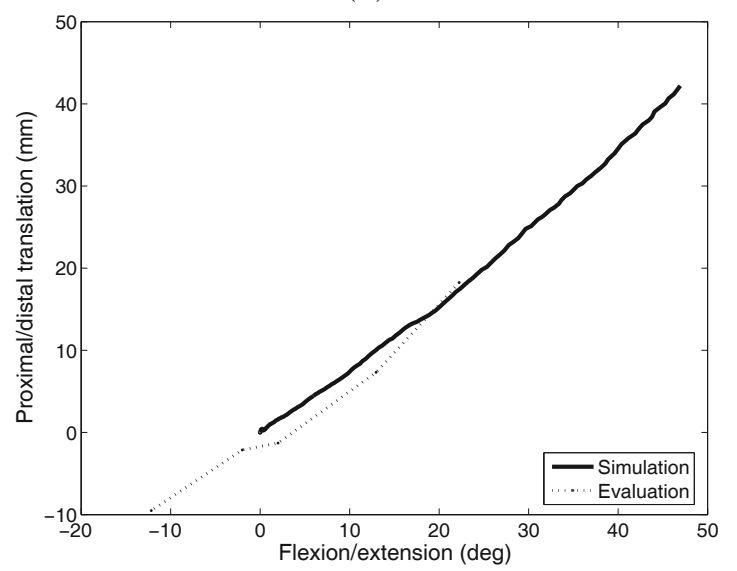

(c)

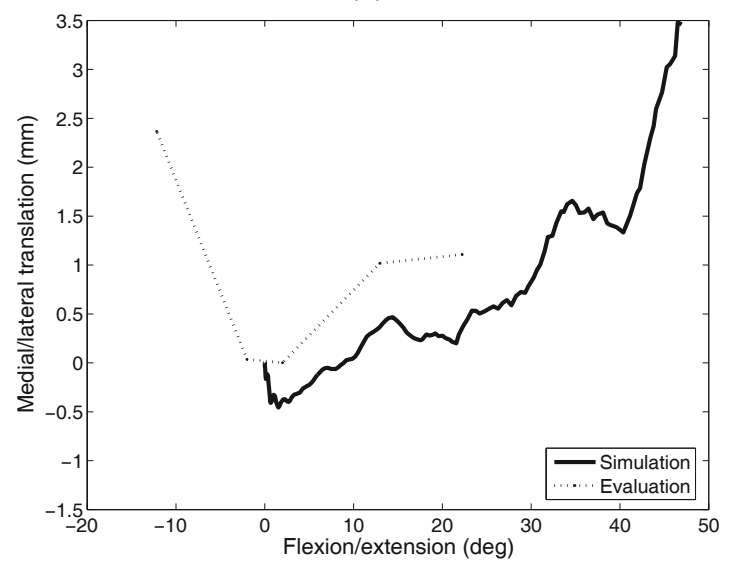

(e)

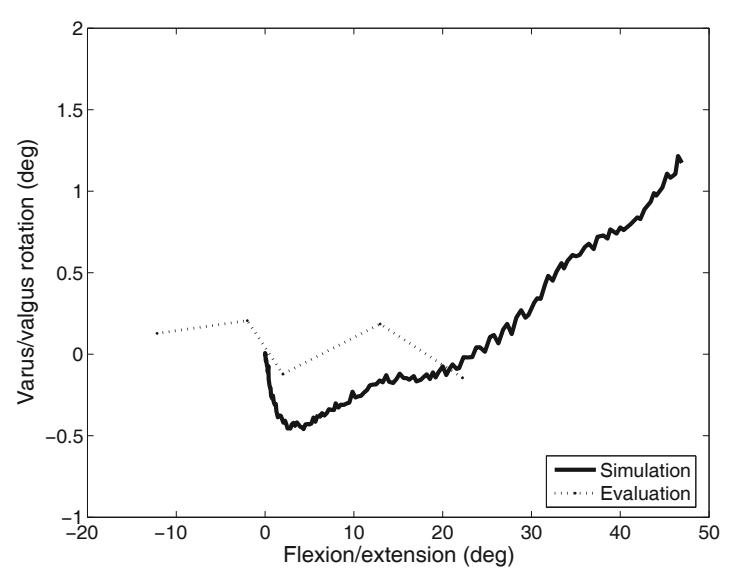

(b)

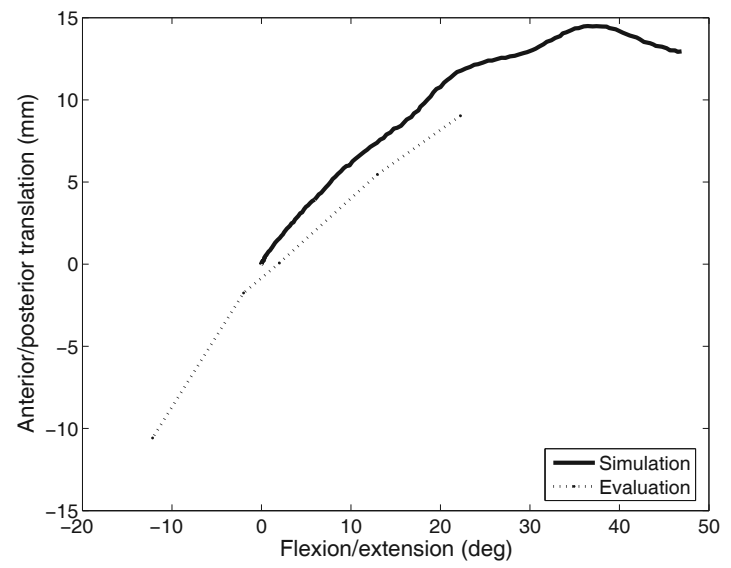

(d)

Figure 7. Simulation (bold) and experimental (dotted) results for patient B: a) internal/external rotation, b) varus/valgus rotation, c) proximal/distal translation, d) anterior/posterior translation, and e) medial/lateral translation. Results are depicted versus flexion angle.

\section{Discussion and conclusions}

The aim of this study was to obtain a patientspecific knee joint kinematics during knee flexion in controlled environment of the MR scanner. Knee flexions from $0^{\circ}$ to around $40^{\circ}-$ $50^{\circ}$ were simulated. This limitation is due to the limited bore of MR scanner constructions (Halloran et al., 2005) and the MR compliant exercise rig. In addition, both devices constrained a permitted patient motion during imaging, nevertheless, only such controlled en- 
vironment makes feasible a credible evaluation of our computer model. Predictions in terms of knee kinematics made by this model were evaluated against experimentally gathered kinematics data from the preselected fixed knee flexion MR imaging.

Figures 6 and 7 depict the obtained motion of the femur bone with respect to the tibia for two patients. Let us asses the Euler angles first. The simulation results for the varus/valgus rotation, depicted in bold (Figures $6 \mathrm{~b}$ and $7 \mathrm{~b}$ ), point out a slight increase and, afterwards, stabilization at around $1^{\circ}$ with the knee flexed by $40^{\circ}$. In contrast, the varus/valgus used for the evaluation (dotted in Figures $6 \mathrm{~b}$ and $7 \mathrm{~b}$ ) alternates around $0^{\circ}$. Due to the controlled testing environment, there was practically no rotation in the varus/valgus. The same is evident in the simulation, where the knee joint appears to be relatively stable in varus/valgus (sideways tilting). A similar phenomenon is shown in the internal/external rotation results (see Figures $6 \mathrm{a}$ and $7 \mathrm{a}$ ). Our simulation points out a slight internal rotation from $0^{\circ}$ to around $4^{\circ}$ at $40^{\circ}$ knee flexion. In contrast, the measured data first demonstrates an initial decrease of $-10^{\circ}$, then a slight increase is noticed to around $1^{\circ}$ at $40^{\circ}$ knee flexion. The measured data clearly indicates, see Figure 7a, the 'screw-home' mechanism (Moglo and Shirazi-Adl, 2005; Piazza and Cavanagh, 2000) in the first $15^{\circ}$ knee flexion (depicted from around $-10^{\circ}$ to around $5^{\circ}$ knee flexion), thereafter appearing to stabilise around $1^{\circ}$. In our computer model the initial knee flexion was determined by the angle used in the static MR acquisition; thus the region in which most of the 'screw-home' would be likely to occur was not modelled. With regard to translations, a good agreement between the model and measured data is ascertained, especially at proximal/distal translation (see Figures $6 \mathrm{c}$ and $7 \mathrm{c})$. In addition, these results point out that the knee joint appears to be relatively stable in medial/lateral translation. A bit larger deviation is detected at anterior/posterior translation, however, we notice a similar tendency of simulation and real data (see Figures $6 \mathrm{~d}$ and $7 \mathrm{~d}$ ). The MRscanned knee flexions were restricted due to the MR compliant exercise rig, while our model imposes no extra restrictions. This caused bigger differences between simulation and experiment at large flexion angles.
The accuracy of the final patient-specific model is critical for predicting patient-specific kinematics. In addition, this accuracy influences also the evaluation procedure (see subsection 3.5). Therefore, a comparison between the template-based automatically constructed patient 3D models and manually created models was carried out. Manual model construction was supervised by orthopaedic surgeons. A disparity between two 3D models was calculated in two different ways: a) on slice-to-slice basis by using mean absolute distance (MAD) and b) on surface (volume) basis by using spherical distance (SD). The average MAD calculated for major knee structures is $0.52 \mathrm{~mm}(\mathrm{std}=0.34$ $\mathrm{mm}$ ), with minimum and maximum being 0.28 $\mathrm{mm}$ and $0.75 \mathrm{~mm}$, respectively. On the other side, the average SD is $1.69 \mathrm{~mm}(\mathrm{std}=3.21$ $\mathrm{mm}$ ), with minimum and maximum being 1.18 $\mathrm{mm}$ and $4.91 \mathrm{~mm}$, respectively (Simbio project, 2003; Heric and Potocnik, 2006). From these results we deduce the template matched patientspecific model creation process is of sufficient quality. If gross inaccuracies in the model appeared, a manual patient-specific model correction should be applied.

Reference data used in this study were measured non-invasively and without radiation during controlled experiment. The accuracy of these measurements is influenced by the accuracy of the $3 \mathrm{D}$ reconstruction procedure (see above) and rigid registration method used when aligning sparse fixed-flexion volume with static volume. Efficiency of the applied rigid registration method was tested on artificially generated knee joint data. This assessing pointed out that the applied registration procedure was up to $\pm 1^{\circ}$ accurate in rotation and up to $\pm 0.2 \mathrm{~mm}$ in translation (Simbio project, 2003). From these findings we estimate that the error in our measurement procedure could be in the worst case up to $2^{\circ}$ by rotation and up to $2 \mathrm{~mm}$ by translation. However, it should be emphasized that measurements data were acquired in a controlled experiment where this error is essentially smaller.

Patient-specific 3D meshes could be automatically generated also directly from patient MRI data without non-rigid registration and template mesh, for example by using methods like Delaunay tessellation or recursive spatial decomposition in the geometrical domain and similar methods. However, by testing such methods 
we found out that obtained patient-specific FE meshes did not provide smooth surface representations. Surfaces contained so called "terracing artefacts" (jagged edges), which resulted from pixelation of the medical imaging data. This problem could be solved only partially by shifting some meshes' nodes. Namely, smooth object boundaries (surfaces) are highly important in non-linear FE analyses. For example, to successfully run a simulation where two curved surfaces move over each other, such as is necessary for simulating knee joint kinematics, both surfaces must be smooth and without notable discontinuities.

In the sequel, this work is compared to similar, previously published works. A very similar experiment was done in (Patel et al., 2004), where the kinematics of constant weight loaded knee during knee flexion from $0^{\circ}$ to $60^{\circ}$ was studied. The work in (Patel et al., 2004) was focused just on motion analysis with no modelling included. The reported measured knee kinematics is in concordance with our results. The tibialfemoral rotations and translations during knee flexion were also studied in (Moglo and ShiraziAdl, 2005; Beillas et al., 2004; in Jan et al., 2002 just rotations). Reported results point out a big similarity with our results. In (Beillas et al., 2004), the root mean square errors between computer model and simulation were around $1.4^{\circ}$ by rotations and around $1 \mathrm{~mm}$ by translations, which is in the same quality class as ours (however, for a slightly different experiment).

The idea of observing or modelling knee joint kinematics while a knee is slightly loaded is not new. To date, this load is either constant as in (Rothe et al., 2004), or variable according to a predefined function at some intervals as in (Godest et al., 2002). Our approach is different to an extent that 'in vivo' acquired load data can be used at the modelling phase. Therefore, a special system was developed for force measurements in the magnetic field as explained in subsection 2.2.

Our modelling and knee-kinematics assessment procedure is fully non-invasive, which makes it perfectly suitable for clinical practice. The described knee joint computer model has not been used in daily clinical practice yet. For such usage, this model needs to be slightly refined and should undergo more thorough evaluation, e.g. by augmenting the evaluation process by other major knee joint structures like the patella bone and menisci. The most important research directions could be the development of a model for capturing fine kinematics, refining meniscal motion, and study the influences of all patientspecific parameters.

Finally, this model could have a significant impact on planning patient-specific operative interventions. It could be especially advantageous in situations where postoperative knee joint stability and functionality are not obvious immediately.

\section{Acknowledgments}

The authors gratefully acknowledge the indispensable contributions of Prof. Dr. David Barber, Dr. Steve Wood, and Dr. Avril McCarthy from the Department of Medical Physics and Clinical Engineering, University of Sheffield, England, whose suggestions and help enabled the origins of this work. This work was supported by the European funding within the 5th Framework project entitled 'SimBio' (Contract No. IST-1999-10378).

\section{References}

[1] E. M. Abdel-Rahman, M. S. Hefzy, Threedimensional dynamic behaviour of the human knee joint under impact loading. Medical Engineering \& Physics, 20 (1998), 276-292.

[2] Y. BEI, B. J. FREGLY, Multibody dynamic simulation of knee contact mechanics. Medical Engineering \& Physics, 26 (2004), 777-789.

[3] P. Beillas, G. Papaioannou, S. Tashman, K. H. YANG, A new method to investigate in-vivo knee behavior using a finite element model of the lower limb. Journal of Biomechanics, 37 (2004), 10191030.

[4] T. L. H. DONAHUE, M. L. Hull, M. M. RASHID, C. R. JACOBS, How the stiffness of meniscal attachments and meniscal material properties affect tibio-femoral contact pressure computed using a validated finite element model of the human knee joint. Journal of Biomechanics, 36 (2003), 19-34.

[5] D. Donlagic, B. Culshaw, Propagation of the fundamental mode in curved graded index multimode fiber and its application in sensor systems. Journal of lightwave technologies, 18(3) (2000), 334-342. 
[6] M. A. R. Freeman, V. Piskernikova, The movement of the normal tibio-femoral joint. Journal of Biomechanics, 38 (2005), 197-208.

[7] W. A. Gambling, H. Matsumura, C. M. RaGDALE, R. A. SAMmUT, Measurement of radiation loss in curved singlemode fibres. Microwaves, optics and acoustics, 2(4) (1978), 134-140.

[8] R. C. Gauthier, C. Ross, Theoretical and experimental consideration for single-mode fibre optic bend-type sensors. Applied optics, 36(25) (1997), 6264-6273.

[9] A. C. Godest, M. Beaugonin, E. Haugh, M. TayLOR, P. J. GREGSON, Simulation of a knee joint replacement during a gait cycle using explicit finite element analysis. Journal of Biomechanics, 35 (2002), 267-275.

[10] J. P. Halloran, A. J. Petrella, P. J. RullkoetTER, Explicit finite element modelling of total knee replacement mechanics. Journal of Biomechanics, 38 (2005), 323-331.

[11] D. Heric, B. PotocnIK, Objective assessment of image segmentation algorithms. Electrotechnical Review, 74(1-2) (2007), 13-18.

[12] S. V. S. Jan, P. Salvia, I. Hilal, V. Sholukha, M. ROOZE, G. ClAPWORTHY, Registration of 6-DOFs electrogoniometry and CT medical imaging for 3D joint modelling. Journal of Biomechanics, 35 (2002), 1475-1484.

[13] M. LAASANEN, Development and validation of mechano-acoustic techniques and instrument for evaluation of articular cartilage. PhD Thesis, Faculty of Natural and Environmental Sciences, University of Kuopio, 2003.

[14] K. E. Moglo, A. ShIRAZI-ADL, Cruciate coupling and screw-home mechanism in passive knee joint during extension-flexion. Journal of Biomechanics, 38(5) (2005), 1075-1083.

[15] V. V. PATEL, K. HALl, M. RiES, J. LOTZ, E. OZHINSKY, C. LINDSEY, Y. LU, S. MAJUMDAR, A threedimensional MRI analysis of knee kinematics. Journal of Orthopaedic Research, 22 (2004), 283-292.

[16] J. M. T. Penrose, G. M. Holt, M. Beaugonin, D. R. HosE, Developement of an accurate threedimensional finite element knee model. Computer Methods in Biomechanics and Biomedical Engineering, 5(4) (2002), 291-300.

[17] S. J. PiazZA, P. R. CAVANAGH, Measurement of the screw-home motion of the knee is sensitive to errors in axis alignment. Journal of Biomechanics, 33 (2000), 1029-1034.

[18] B. Potocnik, D. Heric, D. Zazula, B. Cigale, D. BERNAD, T. TOMAŽIČ, Construction of patient specific virtual models of medical phenomena. Informatica, 29(2) (2005), 209-218.

[19] W. H. Press, S. A. Teukolsky, W. T. Vetterling, B. P. FLANNERY, Numerical Recipes in $C$ : The art of scientific computing. Cambridge University Press, Cambridge, 1992.
[20] R. EISENhart-Rothe, M. Siebert, C. BRINGMANN, T. VoGL, K.-H. ENGLMEIER, H. GRAICHEN, A new in-vivo technique for determination of 3D kinematics and contact areas of the patello-femoral and tibio-femoral joint. Journal of Biomechanics, 37 (2004), 927-934.

[21] P. J. Rowe, C. M. Myles, C. Walker, R. Nutton, Knee joint kinematics in gait and other functional activities measured using flexible electrogoniometry: how much knee motion is sufficient for normal daily life?. Gait and Posture, 12 (2000), 143-155.

[22] N. B. Schuler, M. J. Bey, J. T. Shearn, D. L. BUTLER, Evaluation of an electromagnetic position tracking device for measuring in-vivo, dynamic joint kinematics. Journal of Biomechanics, 38(10) (2005), 2113-2117.

[23] A. B. Sharma, A.-H. Al-Ani, S. J. Halme, Constant-curvature loss in monomode fibres: an experimental investigation. Applied optics, 23(19) (1984), 3297-3301.

[24] SIMBIO PROJECT, 5th framework programme, no. IST-1999-10378, http: //www . simbio.de, 2003.

[25] V. M. ZATSIORSKy, V. N. SeluyanOV, Estimation of the mass and inertia characteristics of the human body by means of the best predictive regression equations. In Biomechanics IX-B (D. A. Winter, R. W. Norman, R. P. Wells, K. C. Hayes, A. E. Patla, Ed.), (1985) pp. 233-239.

[26] X. Zhou, L. F. Draganich, F. Amirouche, A dynamic model for simulating a trip and fall during gait. Medical Engineering \& Physics, 24 (2002), 121-127.

[27] S. Wood, D. C. Barber, A. D. MCCARThy, D. Chan, I. D. Wilkinson, G. DARWENT, D. R. HOSE, A novel image registration application for the invivo quantification of joint kinematics. Presented at the Proceedings of Medical Image Understanding and Analysis, (2002) University of Portsmouth, Portsmouth.

Received: October, 2007 Revised: May, 2008 Accepted: May, 2008

Contact address:

Božidar Potočnik Faculty of Electrical Engineering and Computer Science University of Maribor Smetanova 17, 2000 Maribor, Slovenia Tel.: +38622207484 Fax.: +38622207272 e-mail: bozo.potocnik@uni-mb.si

BOŽIDAR POTOČNIK is an Associate Professor at the Faculty of Electrical Engineering and Computer Science, Maribor. His research interests are segmentation algorithms at computer image processing, pattern recognition, biomedicine, cognitive vision, and machine learning. 
DAMJAn ZaZUla is a Professor at the Faculty of Electrical Engineering and Computer Science, Maribor. His main research interests are compound signal decomposition, biomedical imaging, and virtual training tools.

BORIS CIGALE is a teaching assistant at the Faculty of Electrical Engineering and Computer Science in Maribor. He received his DSc. degree in 2007. His main research interests include image processing of medical images.

DUŠAN HERIC is a teaching assistant at the Faculty of Electrical Engineering and Computer Science in Maribor. He received his DSc. degree in 2007. His main research interests are wavelet transformation-adapted analysis and synthesis, active contours, image registration, edge, and contour line detection.

EDVARD CIBULA received his Ph.D. degree in electrical engineering from the University of Maribor, Slovenia in 2005. His research interests include fiber optic sensors, smart structures and MOEMS technologies.

TOMAŽ TOMAŽIČ is a Senior Supervising Surgeon and Assistant Head of the Clinical Orthopedic Department in the Clinical Centre Maribor. He received his PhD degree in 1991 from the Ruprecht Karls University of Heidelberg, the Diploma in Orthopedic Surgery from the Medical Faculty of the University of Vienna in 1994, and from the Medical Faculty of the University of Ljubljana in 1995. His work and current research is focused on operative treatment of joint and spine disorders, and kinematics disorders of the locomotor system. 